\title{
Absorption recovery in strongly saturated quantum-well electroabsorption modulators
}

\author{
Højfeldt, Sune; Romstad, F.; Mørk, Jesper
}

Published in:

I E E E Photonics Technology Letters

Link to article, DOI:

10.1109/LPT.2003.809986

Publication date:

2003

Document Version

Publisher's PDF, also known as Version of record

Link back to DTU Orbit

Citation (APA):

Højfeldt, S., Romstad, F., \& Mørk, J. (2003). Absorption recovery in strongly saturated quantum-well electroabsorption modulators. I E E E Photonics Technology Letters, 15(5), 676-678.

https://doi.org/10.1109/LPT.2003.809986

\section{General rights}

Copyright and moral rights for the publications made accessible in the public portal are retained by the authors and/or other copyright owners and it is a condition of accessing publications that users recognise and abide by the legal requirements associated with these rights.

- Users may download and print one copy of any publication from the public portal for the purpose of private study or research.

- You may not further distribute the material or use it for any profit-making activity or commercial gain

- You may freely distribute the URL identifying the publication in the public portal

If you believe that this document breaches copyright please contact us providing details, and we will remove access to the work immediately and investigate your claim. 


\title{
Absorption Recovery in Strongly Saturated Quantum-Well Electroabsorption Modulators
}

\author{
Sune Højfeldt, Francis Romstad, and Jesper Mørk
}

\begin{abstract}
We observe experimentally that a quantum-well electroabsorption modulator, when strongly saturated by a highly energetic optical pulse, may exhibit an absorption recovery time much longer than for excitation with a low-energy pulse. Using a comprehensive drift-diffusion type model, we are able to explain this effect theoretically. The prolongation of the absorption recovery is induced by carrier distribution effects, not by field-induced changes in the dynamical transport parameters such as the time carriers take to escape from the wells.
\end{abstract}

Index Terms-Absorption recovery, all-optical signal processing, drift-diffusion model, electroabsorption modulator (EAM), InGaAsP, optical communication, semiconductor devices, sweep-out dynamics.

\section{INTRODUCTION}

D IFFERENT TYPES of components for 40-Gb/s systems as well as for next-generation all-optical systems are currently being evaluated in the laboratories. The electroabsorption modulator (EAM) has been used for pulse generation [1] as well as for a number of all-optical functions, such as demultiplexing, wavelength conversion, and signal regeneration [2], [3], and it may very well become an important part of both $40-\mathrm{Gb} / \mathrm{s}$ systems and future all-optical systems. All-optical functions are performed with EAMs by bleaching the absorption through optical excitation of carriers.

Typically, a component designed for one function will not be optimized or even suitable at all for realizing other functions. Pulse generation and all-optical wavelength conversion, for instance, require components that have quite different designs. An understanding of the detailed dynamics in a specific type of component can support the design of devices that are tailored for these different functions.

In an EAM, the sweep-out of carriers from the active region is one of the main processes that determine the device speed. The carrier sweep-out process is affected when carriers are excited in the device. The excited carriers modify the escape times from the wells, the carrier drift velocities, and other dynamical parameters. When the density of photoexcited carriers is low, these changes will be unimportant. At higher densities, changes in the aforementioned parameters can significantly affect the sweep-out. Especially, field-induced changes in the es-

Manuscript received October 18, 2002; revised January 23, 2003. This work was supported by the Danish Technical Research Council (STVF) through the SCOOP Program. The device was provided by K. Yvind (COM) and J. Hanberg (GiGA - an Intel Company).

The authors are with COM, Technical University of Denmark, Kgs. Lyngby DK-2800, Denmark (e-mail: sh@com.dtu.dk; jm@com.dtu.dk; fr@com.dtu.dk).

Digital Object Identifier 10.1109/LPT.2003.809986 cape times of carriers out of the wells can cause a prolongation of the carrier sweep-out time [4] and is typically the main source for prolongation of the sweep-out time. This is one reason why escape from quantum wells (QWs) has been the subject of many papers that discuss the absorption recovery in EAM-type structures, e.g., [4] and [5]. As an example, a fast modulator with a sweep-out time of, say, 10 ps at low excitation energies can exhibit a sweep-out time of some tens of picoseconds after excitation by a pulse with a significant energy. Field-induced changes in the escape times from the wells would account for most of the increase, whereas field-induced changes in the drift velocities would account for just a few picoseconds, even though the field might become fully screened.

In this letter, we investigate the sweep-out after excitation of very high densities of carriers. In this case, a completely different effect can cause a very significant increase in the carrier sweep-out time, much beyond what the field-induced changes in the dynamical parameters might be responsible for. Using a comprehensive drift-diffusion type model, we are able to explain our measurements. The prolongation is due to charge-distribution effects, which initially suppress carrier transport after excitation.

\section{MODEL}

The carrier sweep-out dynamics is modeled using a drift-diffusion type model similar to those used to model semiconductor lasers [6], [7]. This involves solving Poisson's equation, the continuity equations, and Schrödinger's equation self-consistently. Being very similar to these models, only the main lines important for the discussion in this letter will be drawn up.

To describe the escape time of carriers out of a QW, the expression found by Schneider and von Klitzing [5] is used. For electrons

$$
\tau_{e, 2 \mathrm{D}}=\left(\frac{2 \pi m_{e, \|} L_{\mathrm{QW}}^{2}}{k_{\mathrm{B}} T}\right)^{1 / 2} \exp \left[\frac{E_{\mathrm{b}}-E_{1}}{k_{\mathrm{B}} T}\right]
$$

with a similar expression for holes. In this expression, $m_{e, \|}$ is the in-plane mass of electrons in the well, and $L_{\mathrm{QW}}$ is the width of the well. $E_{1}$ is the energy of the lowest-lying quasibound state. The well states are found by solving the single-particle Schrödinger equation in the envelope approximation. The Eigenstates found by solving the Schrödinger equation are also used to calculate the absorption spectrum; the valence bands are assumed to be uncoupled, and a quadratic dispersion is used in the directions parallel to the $\mathrm{QW}$ layers.

The thermionic emission model is used to describe the current across heterointerfaces [8]. For boundary conditions, we assume 


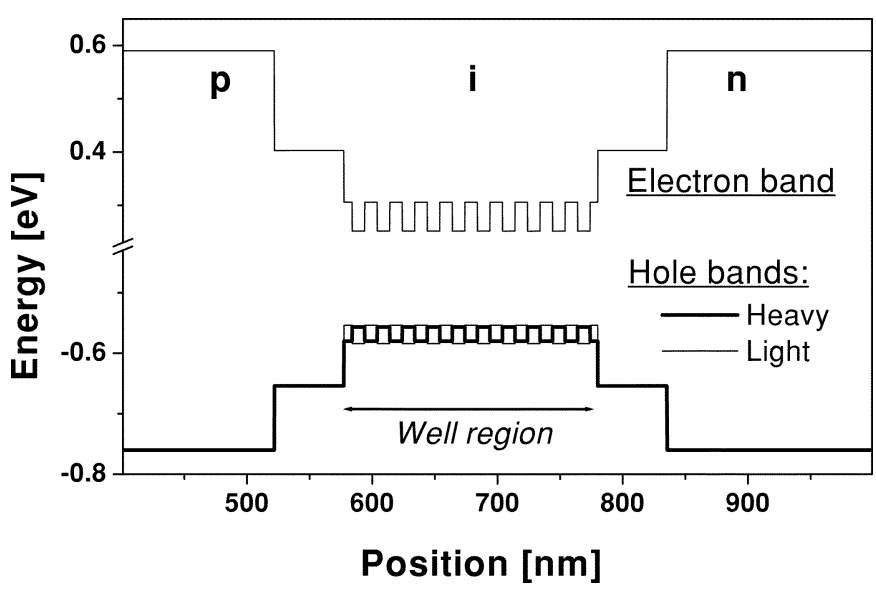

Fig. 1. Banddiagram of the structure under investigation. The diagram also defines what we refer to as the well region.

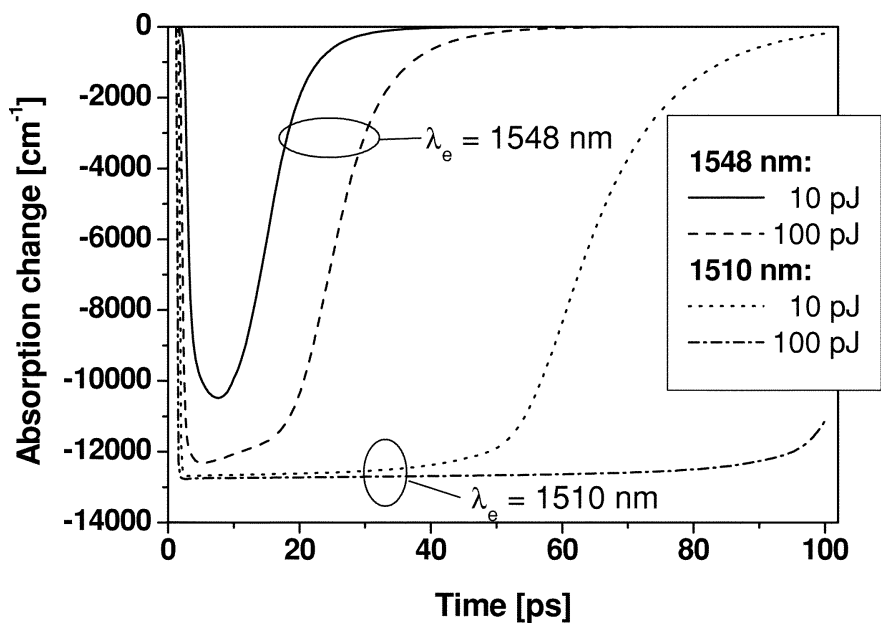

Fig. 2. Calculated absorption recovery after saturation by a short pulse. The recovery is shown for two different excitation wavelengths $\left(\lambda_{\mathrm{e}}\right)$ and two different pulse energies. The excitation pulse is $1 \mathrm{ps}$ wide and centered at $2.5 \mathrm{ps}$.

that the electrostatic voltage from contact to contact is constant in time.

The model does not take propagation effects into account.

\section{RESULTS}

We study an InGaAsP p-i-n structure with ten QW. Its banddiagram is shown in Fig. 1. The structure is biased at $-1 \mathrm{~V}$, where the nominal bandgap is at $1528 \mathrm{~nm}$. The absorption is probed at $1520 \mathrm{~nm}$. We investigate this structure because it has particularly shallow wells, from which carriers escape very quickly. Before optical excitation, the escape time for electrons is $110 \mathrm{fs}$ and the escape time for holes is $70 \mathrm{fs}$. Even with a completely screened field, the escape times do not exceed 450 fs. Thus, field-induced changes in the escape times are of little importance for the sweep-out time for this structure.

Fig. 2 shows the calculated absorption recovery for two different excitation energies, 10 and $100 \mathrm{pJ}$, and two different excitation wavelengths, $\lambda_{\mathrm{e}}=1510 \mathrm{~nm}$ and $\lambda_{\mathrm{e}}=1548 \mathrm{~nm}$. The absorption is higher at $\lambda_{\mathrm{e}}=1510 \mathrm{~nm}$ than at $\lambda_{\mathrm{e}}=1548 \mathrm{~nm}$, and a considerably higher density of carriers results from excitation at the shorter wavelength. The four curves in Fig. 2, thus, represent different densities of excited carriers.

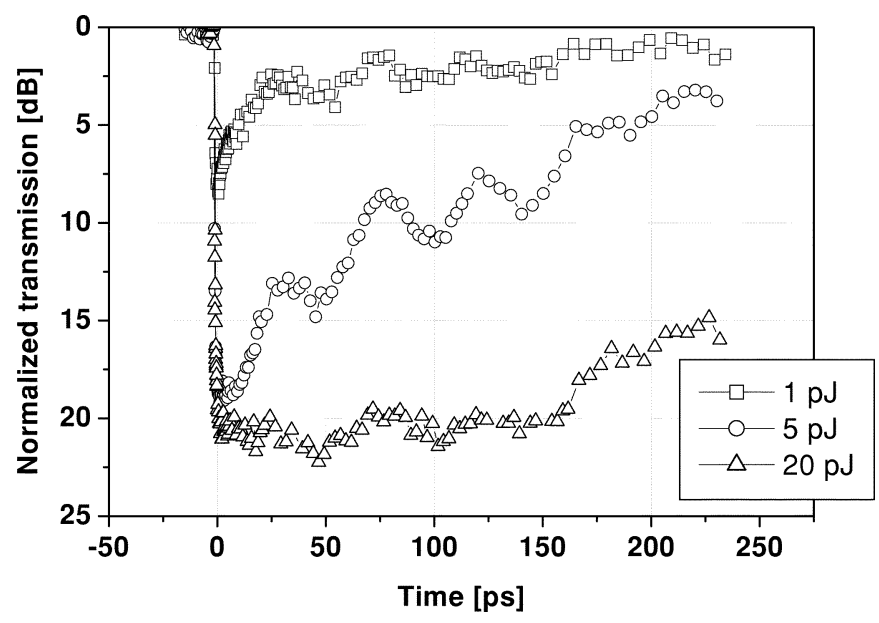

Fig. 3. Normalized transmission measured in a pump-and-probe experiment using 200-fs wide pulses at $1520 \mathrm{~nm}$, centered at $0 \mathrm{ps}$.

In the 10-pJ $\lambda_{\mathrm{e}}=1548$-nm case, the recovery is fast, below 20 ps. In this case, only field-induced changes in the dynamical parameters affect the sweep-out time, which is just a few picoseconds longer than the low-energy sweep-out time. For excitation at the shorter wavelength and with an energy of $100 \mathrm{pJ}$, the recovery time is longer than $100 \mathrm{ps}$, several orders of magnitude longer than the escape times from the wells. It is, thus, clear that field-induced changes in the escape times are not responsible for the prolongation.

Measurements of the absorption recovery as function of excitation pulse energy were made on a device with the same nominal epitaxial structure as that in Fig. 1. The device is a $250-\mu \mathrm{m}$-long ridge waveguide structure that was biased at $-3 \mathrm{~V}$ in the experiments. The absorption was pumped and probed at $1520 \mathrm{~nm}$. The measurements are shown in Fig. 3. The origin of the oscillations seen in Fig. 3 has not been established, but there are indications that they are caused by the electrical circuit.

The measurements and calculations show good qualitative agreement - a fast recovery for low excitation pulse energies, and a prolonged recovery at high energies. The measured recovery times are similar for excitation pulse energies of 1 and $5 \mathrm{pJ}$. However, at $20 \mathrm{pJ}$, the recovery takes much longer, more than 200 ps.

The long recovery time is due to a special physical situation that comes about at very high carrier densities. Upon excitation, the field in the well region will be completely screened, and a quasi-equilibrium is established in which the sign of the charge density changes between positive and negative across the well region. This happens because of an asymmetry between the densities of confined electrons and confined holes. It results in a field across the well region which changes sign near the middle of each well and each barrier, as shown in Fig. 4. The field forces bulk carriers (i.e., carriers in quasi-continuum states) toward the zero-field points, electrons toward the zero-field point in the barriers, and holes toward the zero-field point in the wells. From an energy perspective, the change in the field direction corresponds to a potential well. The depths of the potential wells increase with the density of excited carriers and thus with excitation energy. Only near the edge of the well region, where the field is high, are carriers accelerated by the field. Due to the potential 


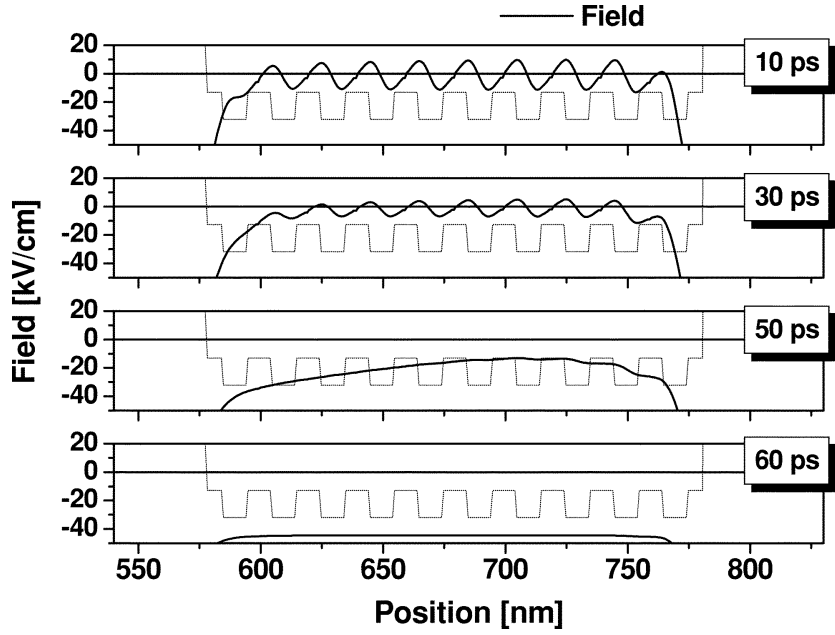

Fig. 4. Calculated field in the well region at different times after excitation at $1510 \mathrm{~nm}$ by a pulse with a high energy, $10 \mathrm{pJ}$. After the excitation, the field remains completely screened for some time due to the carriers forming potential wells that impede carrier transport. For reference, the banddiagram is also shown

wells, carriers are removed only very slowly from the well region through a process where they diffuse out and are swept away by the field that exists outside the well region. However, the potential wells persist for a long time, as quantified in Fig. 2. With time, the depths of the potential wells are reduced, and as they are eliminated the transport becomes dominated by drift and the recovery will, therefore, proceed fast. As mentioned above, the potential well depths increase with the density of excited carriers. Hence, the plateau part of the recovery, where the absorption is completely saturated, is longer for higher excitation energies. However, once the actual recovery sets in, it proceeds in almost the same fashion irrespective of the excitation pulse energy, as Fig. 2 shows.

The calculations and the measurements presented in this letter should not be compared quantitatively. The emphasis is entirely on the qualitative behavior, namely that both calculations and measurements display, to the same order of magnitude, a significantly prolonged absorption recovery when the excitation pulse energy is very high. There are a number of reasons why a quantitative comparison should not be attempted. We mentioned earlier that propagation effects are not included in the calculations. In the measurements, these effects are important at excitation energies 1 and $5 \mathrm{pJ}$. The 20-pJ pulse, however, saturates the device to such a degree that we can assume that the absorption coefficient is uniform through the length of the device, in which case we could, in principle, directly compare calculations and measurement (the total absorption in decibels will be proportional to the absorption coefficient). However, even in this case, a direct comparison between calculations and measurements is made difficult by the uncertainties in the device and material parameters. The banddiagram in Fig. 1 is the nominal epitaxial structure but is uncertain with respect to the exact $\mathrm{QW}$ widths, band offsets, etc. Also, the values of parameters such as carrier masses and mobilities are typical values taken from the literature and they might well be different from those that charac- terize our sample. As an example, general measurements of the mobility in quaternaries and its dependence on the electric field do not, to our knowledge, exist. The mobility is usually characterized only for lattice-matched material. Our structure is highly strained in the QW region. Other effects, such as carrier heating, might also play a role [9]. Finally, we emphasize that the prolongation of the recovery is observed in a large parameter space and that the uncertainties described above will not lead to qualitative changes in the results.

\section{CONCLUSION}

We have modeled the absorption recovery in an EAM after excitation of very high carrier densities. In qualitative correspondence with our measurements, we find that the absorption recovery time can be much longer than at moderate carrier densities.

The prolongation is caused by a combination of two effects: an initially diffusion-dominated transport—because of the high carrier density - and the formation of potential wells in the well region that suppress transport in this region. When the carrier density in the well region becomes low enough that the field starts to recover and the potential wells are eliminated, the recovery will proceed fast, in a fashion that is almost independent of the initial excitation energy.

Simple models that do not take the carrier distribution and field-induced changes in the dynamical parameters into account would fail to account for this behavior, illustrating that comprehensive models are required for understanding the complicated dynamics in EAMs.

\section{REFERENCES}

[1] T. Ido, S. Tanaka, M. Suzuki, and H. Inoue, "MQW electroabsorption optical modulator for $40 \mathrm{Gbit} / \mathrm{s}$ modulation," Electron. Lett., vol. 31, no. 24, pp. 2124-2125, 1995.

[2] T. Otani, T. Miyazaki, and S. Yamamoto, " $40 \mathrm{Gbit} / \mathrm{s}$ signal transmission using optical $3 \mathrm{R}$ regenerator based on electroabsorption modulator," in Proc. OFC 2000, vol. 3, 2000, pp. 226-228.

[3] L. K. Oxenløwe, E. Hilliger, A. Tersigni, A. M. Nik, S. Højfeldt, F. Romstad, K. Yvind, P. M. W. Skovgaard, K. Hoppe, and J. Hanberg, "All-optical demultiplexing and wavelength conversion in an electroabsorption modulator," Tech. Dig. ECOC, vol. 4, pp. 604-605, 2001.

[4] A. M. Fox, D. A. B. Miller, G. Livescu, J. E. Cunningham, and Jan W. Y, "Quantum well carrier sweep out: Relation to electroabsorption and excitonic saturation," IEEE J. Quantum Electron., vol. 27, pp. 2281-2295, Oct. 1991.

[5] H. Schneider and K. von Klitzing, "Thermionic emission and Gaussian transport of holes in a GaAs $/ \mathrm{Al}_{x} \mathrm{Ga}_{1-x}$ As multiple-quantum-well structure," Phys. Rev. B, vol. 38, no. 9, pp. 6160-6165, 1988.

[6] N. Tessler, "Dynamic properties of inverted quantum well laser structure," Ph.D. dissertation, Technion, Haifa, Israel, 1995.

[7] M. Grupen and K. Hess, "Simulation of carrier transport and nonlinearities in quantum-well laser diodes," IEEE J. Quantum Electron., vol. 34, pp. 120-140, Jan. 1998.

[8] M. Grupen, K. Hess, and G. H. Song, "Simulation of transport over heterojunctions," in Proc. IEEE Electron Devices Soc. 4th Int. Conf. Simul. Semiconductor Device Process., vol. 4, 1991, pp. 303-311.

[9] A. V. Uskov, J. R. Karin, J. E. Bowers, J. G. McInerney, J. L. Bihan, and R. Nagarajan, "Effects of carrier cooling and carrier heating in saturation dynamics and pulse propagation through bulk semiconductor absorbers," IEEE J. Quantum Electron., vol. 34, pp. 2162-2171, Nov. 1998. 